\title{
Mini-Review on Mini-Black Holes from the Mini-Big Bang
}

\author{
M. Bleicher ${ }^{1,2}$ and P. Nicolini ${ }^{1,2, \star}$ \\ 1 Frankfurt Institute for Advanced Studies, Ruth-Moufang-Str. 1, D-60438 Frankfurt am Main, Germany \\ 2 Institut für Theoretische Physik, Johann Wolfgang Goethe-Universität, Frankfurt am Main, Germany
}

The dates of receipt and acceptance should be inserted later

Key words Black Hole Physics, Extra-Dimensions, Terascale Quantum Gravity

\begin{abstract}
We review the basic ideas about man-made quantum mechanical black holes. We start by an overview of the proposed attempts to circumvent the hierarchy problem. We study the phenomenological implications of a strong gravity regime at the terascale and we focus on the issue of microscopic black holes. We provide the experimental bounds on relevant quantities as they emerge from major ongoing experiments. The experimental results exclude the production of black holes in collisions up to $8 \mathrm{TeV}$. We provide some possible explanations of such negative results in view of forthcoming investigations.
\end{abstract}

(c) 2014 WILEY-VCH Verlag GmbH \& Co. KGaA, Weinheim

\section{Introduction}

Gravity is certainly the most commonly known fundamental interaction. In every moment we experience our body weight and we are familiar with the fall of objects. No other fundamental interaction affects everyday life so widely and continuously as gravity. Also the absence of gravity does not come as a surprise. Space explorations made popular this concept, we often call "weightlessness". However, despite the popularity, gravity is probably the less-understood fundamental interaction. Such incomplete understanding is already evident from the more elementary concepts. For instance the gravitational constant, $G=6.67384(80) \times$ $10^{-11} \mathrm{~N}(\mathrm{~m} / \mathrm{kg})^{2}$ (Mohr, Taylor \& Newell 2011), is currently known with an accuracy slightly better than that of the original Cavendish experiment in 1798 (Gillies 1997). The difficulties in performing accurate measurements are explained by the fact that gravity is extraordinarily weak and becomes sizable only if huge bodies are taken into account. Accordingly measurements of gravity become even more complicated at short scales. At typical scales of particle physics gravity is simply negligible. Even for the heaviest elementary particles (e.g. W bosons, top quarks) electromagnetic interactions exceed gravitational forces for something like 32 orders of magnitude.

The reason why gravity is so weak is an open issue that is usually labeled as "hierarchy problem". A possible way to overcome this difficulty is based on the idea that gravity, even if weak at macroscopic scales, might be strong at some minuscule scale. Such a conjecture is admissible because we cannot experimentally measure gravity at scales below some tens of microns (Long et al. 2003; Hoyle et al. 2004, Adelberger et al. 2007, Adelberger et al. 2009). As a result one can contrive a mechanism in order to increase the

^ Corresponding author: e-mail: nicolini@th.physik.uni-frankfurt.de

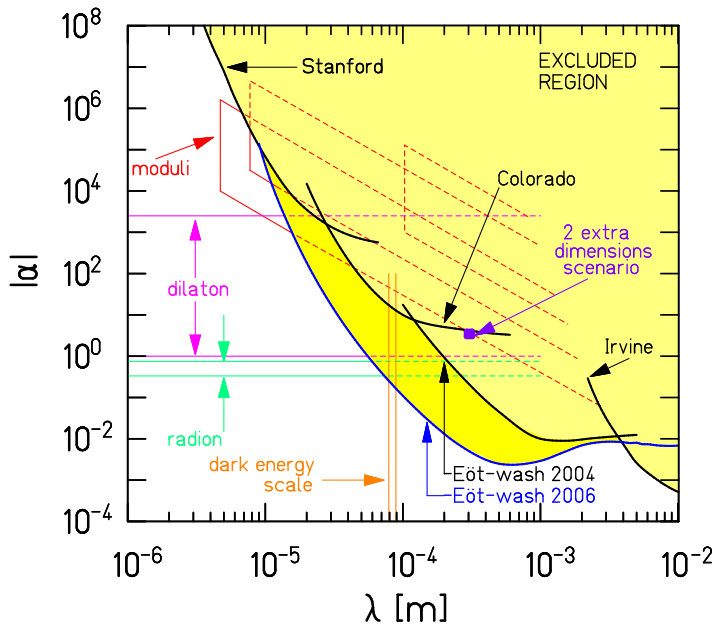

Fig. 1 Deviations from the inverse square law (Long et al. 2003).

strength of gravity, by postulating a different spacetime structure at microscopic scales. This opportunity is offered by the so called "extra-dimensions", i.e., additional spatial dimensions that have to be small enough to be conventionally unobserved. Extra-dimensions are the natural theoretical framework for Superstring theory, Supergravity, M-theory but have a long history dated back to 1921, the year in which the Kaluza-Klein (KK) theory was presented. To solve the hierarchy problem, however, the typical size, $R$, of each additional dimension cannot be arbitrarily small, e.g. $R \sim 1 / m_{\mathrm{P}}$, where $m_{\mathrm{P}} \sim 10^{16} \mathrm{TeV}$ is the Planck mass. Rather we require $R$ to be large enough in order to "lower down" the Planck mass to a new fundamental mass, $M_{\mathrm{F}}$, which has to be above the electroweak scale, $\Lambda_{\mathrm{EW}} \sim 200 \mathrm{GeV}$, but at the reach of (or at least not too far 
from) current particle accelerator energies. Following this reasoning, one would like to place $M_{\mathrm{F}}$ at the terascale, i.e., $M_{\mathrm{F}} \sim 1-10 \mathrm{TeV}$. In addition, to reproduce known results from particle physics, one requires that only gravity can probe extra-dimensions, while conventional Standard Model fields must be constrained on a $(3+1)$-dimensional sub-manifold, called brane1.

\section{Extra-dimensions: why and how}

Along the above line of reasoning, in the late 1990's Antoniadis et al. (1998), Arkani-Hamed, Dimopoulos \& Dvali $(1998 \mathrm{a}, 1998 \mathrm{~b})$ presented the model of large extradimensions (also known as ADD model) as a viable solution to the hierarchy problem. The basic ingredient of such an approach is a $(4+d)$-dimensional bulk spacetime, $\mathcal{M}^{(4+d)}$, that can be factorized as $\mathcal{M}^{(4+\mathrm{d})}=\mathcal{M}^{(4)} \times T^{(d)}$, where $\mathcal{M}^{(4)}$ is the brane and $T^{(d)}$ is a $d$-dimensional torus with radii of size $R$. From the higher-dimensional EinsteinHilbert action, one can obtain a dimensionally reduced action by performing a KK expansion of the graviton field. Since the zero mode is independent of extra-dimensional variables, one can integrate the $d$ additional dimension to obtain (Shifman 2010)

$$
\begin{aligned}
& S_{d+4} \sim M_{\mathrm{F}}^{d+2} \int \mathcal{R} \sqrt{-g} \mathrm{~d}^{4} x \mathrm{~d}^{d} y \\
& \rightarrow \underbrace{M_{\mathrm{F}}^{d+2} R^{d} \int{ }^{(4)} \mathcal{R} \sqrt{-{ }^{(4)} g} \mathrm{~d}^{4} x}_{\text {effective brane action }}+\underbrace{\sum_{k>0}(\ldots)}_{\text {KK excitations }} .
\end{aligned}
$$

The effective 4-dimensional action has to match the standard gravitational action for distances larger than $R$. This matching sets the fundamental scale as $M_{\mathrm{F}} \sim$ $\left(m_{\mathrm{P}}^{2} / R^{d}\right)^{1 /(d+2)}$. Conversely for distances smaller than $R$ one can find compelling deviations to Newton's law

$$
V(r) \sim \frac{1}{M_{\mathrm{F}}^{d+2}} \frac{m_{1} m_{2}}{r^{d+1}}, \quad r<R,
$$

where $m_{1}$ and $m_{2}$ are two test masses at distance $r$. By setting $M_{\mathrm{F}} \sim 1 \mathrm{TeV}$, one obtains the size of extra-dimensions as $R \sim 10^{\frac{32}{d}-19} \mathrm{~m}$. The cases $d=1,2$ are immediately ruled out since they would require too large values for $R$. On the other hand for $d>2$, sub-millimeter measurements of the inverse square law become rather ineffective in setting parameter bounds (see Fig. 1). We remark here that the emission of graviton KK exitations can be constrained with astrophysical tests like the cooling of hot stars e.g. the supernova SN 1987A. Resulting bounds on extradimension turn to be more stringent. The cases $d=1-4$ are ruled out but for $d>4$ the limits are rather loose (Friedland \& Gianotti 2008).

\footnotetext{
1 For sake of completeness, we recall that models with universal extra-dimensions allow the propagation of all fields in the full higherdimensional spacetime (Appelquist, Cheng \& Dobrescu 2001).

2 Antoniadis (1990) first proposed the idea almost a decade earlier.
}

An alternative proposa 3 to the ADD model is offered by the warped extra-dimensions. Following the lines of Randall \& Sundrum (RS) (1999a) $\sqrt{4}$, one postulates the existence of just one extra-dimension to obtain a 5 -dimensional warped anti-deSitter geometry

$$
\mathrm{d} s^{2}=e^{-2 \kappa R|\varphi|} \eta_{\mu \nu} \mathrm{d} x^{\mu} \mathrm{d} x^{\nu}+R^{2} \mathrm{~d} \varphi^{2}
$$

where $\kappa$ is a scale $\sim m_{\mathrm{P}}, x^{\mu}$ are the conventional 4dimensional coordinates, while $0 \leq \varphi \leq \pi$ is the coordinate of the extra-dimension whose size is $\pi R$. At the boundary of the above 5 -dimensional spacetime, there are two branes 5 called Planck (or hidden) brane and $\mathrm{TeV}$ (or visibile) brane, for $\varphi=0, \pi$ respectively. The fundamental mass turns out to be of the order of the Planck masss, $M_{\mathrm{F}} \sim m_{\mathrm{P}}$. However this is not a source of concern because any mass parameter $m_{0}$ will correspond on the visible brane to a physical mass suppressed by the warp factor, $m=e^{-\kappa R \pi} m_{0}$. Contrary to the ADD model, the exponential factor lets us have a large hierarchy of scales without extremely large values of $R$. If $R$ is just few tens of Planck lengths $l_{\mathrm{P}} \equiv 1 / m_{\mathrm{P}} \sim 10^{-35}$ $\mathrm{m}$, the fundamental scale will be lowered down to the $\mathrm{TeV}$ regime on the visible brane. In addition, there are no KK light modes (but only TeV KK modes) and constraints from supernova cooling do not apply.

In conclusion, extra-dimensional models are more effectively tested if one probes small distances by means of high energy physics experiments (Feng 2003).

\section{Black holes at colliders}

Black holes (BHs) are conventionally known as spacetime regions of no escape, delimited by what is technically known as an event horizon. BHs result from solutions of Einstein's field equations and their actual existence is corroborated by the astronomical observations of several candidate BH objects (Celotti, Miller \& Sciama 1999). The size of BHs is set by their gravitational radius $r_{\mathrm{g}} \sim G M_{\mathrm{BH}} / c^{2}$. Depending on their mass, we usually distinguish stellar BHs, intermediate mass BHs and supermassive BHs. The total mass range is rather wide, i.e., $M_{\mathrm{BH}} \sim 10-10^{10} M_{\odot}$ where $M_{\odot} \sim 10^{30} \mathrm{~kg}$ is the solar mass. Accordingly also their formation mechanism is expected to differ drastically. If one considers even smaller masses and radii, i.e., $M_{\mathrm{BH}}<$ $M_{\text {Moon }} \sim 10^{23} \mathrm{~kg}$ and $r_{\mathrm{g}}<0.1 \mathrm{~mm}$, tiny BHs might have formed due to extreme local matter density fluctuations in the primordial Universe (Carr \& Hawking 1974). Alternatively such primordial BHs might have been pair produced from the quantum mechanical decay of the deSitter early Universe (Mann \& Ross 1995, Bousso \& Hawking 1996).

\footnotetext{
${ }^{3}$ For sake of brevity we cannot recall here all alternative mechanisms to tackle the hierarchy problem, which include, among the others, split fermion models Arkani-Hamed \& Schmaltz 2000 Arkani-Hamed, Grossman \& Schmaltz 2000 Mirabelli \& Schmaltz 2000) and un-particle enhancement models (Mureika 2008).

${ }^{4}$ Gogberashvili $(1999,2000,2002)$ proposed an analogue set up in terms of "thin shells".

5 Randall \& Sundrum (1999b) later proposed a single brane model.
} 
The extreme conditions of such scenarios occurring in the early Universe provide some initial clues about how difficult is to artificially produce a microscopic black hole in a particle collision. Following the "hoop conjecture" (Thorne 1972), one finds that the collision energy should exceed $m_{\mathrm{P}}$ to produce a $\mathrm{BH}$, an occurrence that is far above accessible experimental energies. Conversely, if gravity is strong at shortest scales, gravitational collapses become possible at energies at the reach of current accelerators, i.e., $\sim M_{\mathrm{F}}$ (Banks \& Fischler 1999, Bleicher et al. 2002). One may wonder, however, what extra-dimension set up has to be used for a consistent BH description. Technically the ADD model has the advantage of $\mathrm{BH}$ geometries that do not suffer of significant tidal distortions as in warped extradimension models (Maartens 2004). This is a direct consequence of the largeness of extra-dimensions. The size of BHs in the ADD model, $r_{\mathrm{g}} \sim 10^{-19} \ll R$ permits to neglect manifold boundary effects.

The first papers concerning the concrete possibility of producing BHs at the LHC are due to Argyres, Dimopoulos \& March-Russell (1998), Banks \& Fischler (1999), Dimopoulos \& Landsberg (2001) and Giddings and Thomas (2002). Nowadays there exists a huge number of papers, reviews and books about several aspects of the topic, that we cannot address in the present contribution. For an "incomplete" list of suggested readings see (Landsberg 2002, Cavaglià 2003, Kanti 2004, Hossenfelder 2005, Casanova \& Spallucci 2006, Bleicher 2007, Winstanley 2007, Nicolini 2008 Bleicher \& Nicolini 2010; $\quad$ Calmet 2010, Park 2012, Kanti \& Winstanley 2014).

From the static, hyper-spherical BH geometry (Tangherlini 1963), one has

$$
r_{\mathrm{g}}^{d+1}=\left(\frac{1}{M_{\mathrm{F}} \sqrt{\pi}}\right)^{d+1}\left(\frac{M_{\mathrm{BH}}}{M_{\mathrm{F}}}\right)\left[\frac{8 \Gamma\left(\frac{d+3}{2}\right)}{d+2}\right] .
$$

One can see that, for $M_{\mathrm{BH}} \approx M_{\mathrm{F}}$, the gravitational radius $r_{\mathrm{g}} \sim 1 / M_{\mathrm{F}}$ weakly depends on the number of dimensions. In addition such profile of $r_{\mathrm{g}}$ confirms that large extra-dimensions are a crucial ingredient for enhancing the $\mathrm{BH}$ production rate. The decrease of the gravitational scale from $m_{\mathrm{P}}$ to $M_{\mathrm{F}}$ implies an increase of the $\mathrm{BH}$ cross section $\sigma(X X \rightarrow \mathrm{BH}) \sim \pi r_{\mathrm{g}}^{2}$. Despite the intense discussion in the literature about a variety of proposed modifications (Cavaglià 2003), the above black disk profile is widely accepted, at least for collision energies far above $M_{\mathrm{F}}$ (Mureika, Nicolini \& Spallucci 2012). Accordingly the estimated cross section value is $\sigma \sim 400 \mathrm{pb}$. Given the LHC design luminosity $L \sim 10^{38} \mathrm{~m}^{-2} \mathrm{~s}^{-1}$, about a hundred BHs per second would form in particle detectors, or equivalently a billion BHs per year (Hossenfelder et al. 2002, Bleicher et al. 2002).

The life of a $\mathrm{BH}$ in a particle detector is a problem of formidable complication. Mini BHs behave rather differently from their macroscopic counterparts. At microscopic scales quantum mechanical effects cannot be ne- glected. As shown by Hawking (1975), this opens the possibility of tunneling particles through the even horizon, which acts like any other potential barrier. The resulting effect, known as $\mathrm{BH}$ evaporation, is a thermal emission of particles at a temperature $T_{\mathrm{H}} \sim 1 / r_{\mathrm{g}}$, with a consequent $\mathrm{BH}$ decay. In general, the description of these issues would require a quantum theory of gravity, since the spacetime itself is subject to relevant quantum modifications at these scales. For instance string theory encodes modifications related to the idea of non-commutative geometry (Seiberg \& Witten 1999) or the generalized uncertainty principle (Veneziano 1986). Accordingly one can model these characters by an effective implementation in BH spacetimes (Nicolini, Smailagic \& Spallucci 2006, Isi, Mureika \& Nicolini 2013). However, in the transPlanckian regime, i.e., for $M_{\mathrm{BH}} \gg M_{\mathrm{F}}$, the semi-classical approximation of quantum gravity can still be used for drawing scenarios of $\mathrm{BH}$ evaporation.

For pedagogical purposes, one can distinguish four phases of the life of a mini-BH after its formation

i) Balding phase, during which the gauge field hair is shed and the asymmetries removed by gravitational radiation;

ii) Spin-down phase, during which the black hole evaporates through Hawking and Unruh-Starobinskii (Starobinskii 1973, Unruh 1974) radiation, losing mostly angular momentum and mass;

iii) Schwarzschild phase, during which the black hole keeps evaporating via Hawking radiation, but now in a spherical manner;

iv) Planck phase, during which $M_{\mathrm{BH}} \sim T_{\mathrm{H}} \sim M_{\mathrm{F}}$ and quantum gravity effect cannot be neglected.

The destiny of an evaporating BH is uncertain. However there are two prevailing scenarios. The first possibility is that the $\mathrm{BH}$ concludes its life by a non-thermal emission of particles (Emparan, Horowitz \& Myers 2000, Giddings \& Thomas 2002; Calmet, Fragkakis \& Gausmann 2012). Alternatively, the $\mathrm{BH}$ might undergo a cooling down phase towards a zero temperature remnant configuration (Hossenfelder et al. 2003). The latter scenario seems to be a model independent character common to several spacetime models, derived or inspired by quantum gravity considerations (Bonanno \& Reuter 2006, Modesto 2006, Nicolini et al. 2006; Modesto, Moffat \& Nicolini 2011; Nicolini 2012, Isi et al. 2013). If this were the case, the remnant formation would affect the emission spectra 6 any cooling down phase markedly implies an emission of softer particles mostly on the brane (Gingrich 2010, Nicolini \& Winstanley 2011).

The properties of emission spectra are included in the more general framework of the signatures of $\mathrm{BH}$ production. To this purpose we recall that the Hawking emission is not expected to be directly observed in particle

\footnotetext{
${ }^{6}$ Remnants can form also in the absence of a cooling down, like in the case of hot Planckian remnants (Adler, Chen \& Santiago 2001) or following dimensional reduction mechanisms (Mureika 2012 Mureika \& Nicolini 2013,
} 
detectors. Rather one believes that, being mini BHs extremely hot, $T_{\mathrm{H}}>100 \mathrm{GeV}$, the emitted energy can trigger the formation of a photo- and a chromo-sphere, i.e., an electron-positron-photon plasma and a quark-gluon plasma respectively. Such particle atmospheres might result from pair production and bremsstrahlung mechanisms. Accordingly the realistic situation in a particle detector is rather complex: colliding partons are followed by a multiplicity of particles in the case of BH formation. Both QED and QCD drive such process with different critical temperatures, namely $T_{\mathrm{c}}^{\mathrm{QED}} \sim 50 \mathrm{GeV}$ and $T_{\mathrm{c}}^{\mathrm{QCD}} \sim 175 \mathrm{MeV}$ respectively. The discrepancy between the two temperatures explains why the actual $\mathrm{BH}$ emission is dominated by hadrons, which result from parton fragmentation. Specifically, one can estimate that the secondary emission consists of $60 \%$ quarks, $15 \%$ gluons, $10 \%$ leptons, $6 \%$ weak bosons, $5 \%$ neutrinos, $1 \%$ photons and smaller fractions of invisible neutrinos, gravitons as well as new particles around 100 $\mathrm{GeV}$ (Casanova \& Spallucci 2006). In the end, the original Hawking spectrum becomes an effective black body spectrum with a temperature lower than $T_{\mathrm{H}}$. This is simply due to the energy conservation and the mechanisms of particle proliferation that decrease the average energy per particle.

Additional BH signatures consist in events with a reduced visible energy (due to non-detectable gravitational degrees of freedom emitted in the bulk), with exotic particle production, e.g. gluino, squark (Chamblin, Cooper \& Nayak 2004), and deformed hadron spectra at high transverse momentum (Erkoca, Nayak \& Sarcevic 2009). In case of remnant formation, the $\mathrm{BH}$ event would be indirectly recognized in terms of a significant decrease of the total transverse momentum due to the absence of final decay particles (Koch, Bleicher \& Hossenfelder 2005). $\quad$ Alternatively charged BH remnants might be directly detectable by ionization tracks in time projection chambers.

\section{Update on experimental constraints}

Experimental investigations at the LHC aim to set indirect and direct constraints on relevant parameters, e.g. $M_{\mathrm{F}}$, from events signaling the presence of quantum gravity effects. For instance indirect constraints can be obtained from the exchange of gravitons at tree and one-loop level. Direct constraints are obtained by the observation of BHs.

Franceschini et al. (2011) provided a detailed analysis of processes involving graviton exchange at the light of the latest ATLAS and CMS data. Fig. 22 shows the parameter space for the case of 5 extra-dimension. The function $\Lambda / M_{D}$ is plotted versus $M_{D}$, where $\Lambda$ is an ultraviolet cut off and $M_{D}$ denotes the fundamental mass. Physically $\Lambda$ represents the mass of new states associated to any ultraviolet completion of gravity. Despite $\Lambda$ is an unknown parameter, the advent of LHC has further constrained the parameter space with respect to previous analyses based on the LEP

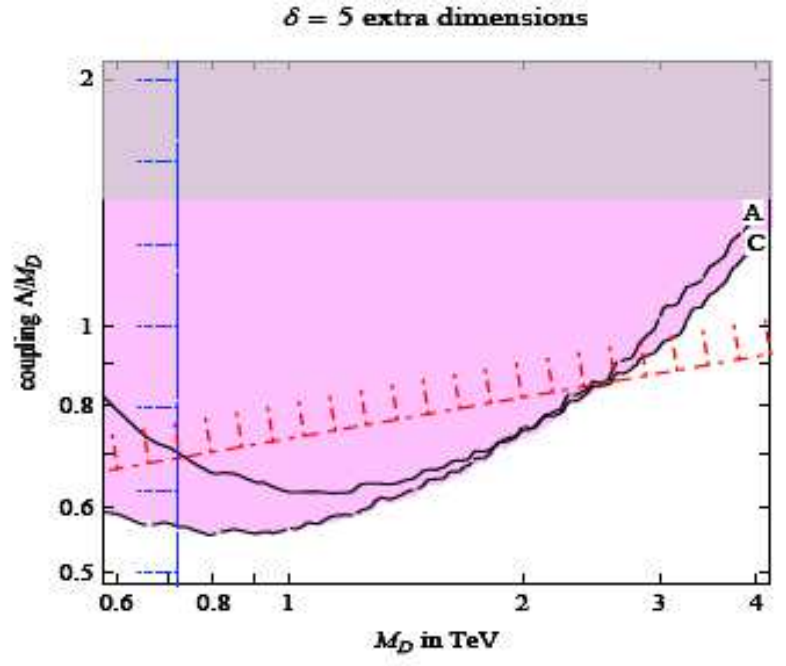

Fig. 2 (Franceschini et al. 2011). The shaded area is the bound from virtual graviton exchange at CMS (continuous line denoted as 'C', data after 36/pb), ATLAS (long-dashed line denoted as 'A', data after 36/pb). Vertical blue line: bound from graviton emission in (Giudice \& Sturmia 2003). Red line: Naive Dimensional Analysis (NDA) estimate of LEP bound from loop graviton exchange. Upper shading: NDA estimate of the nonperturbative region.

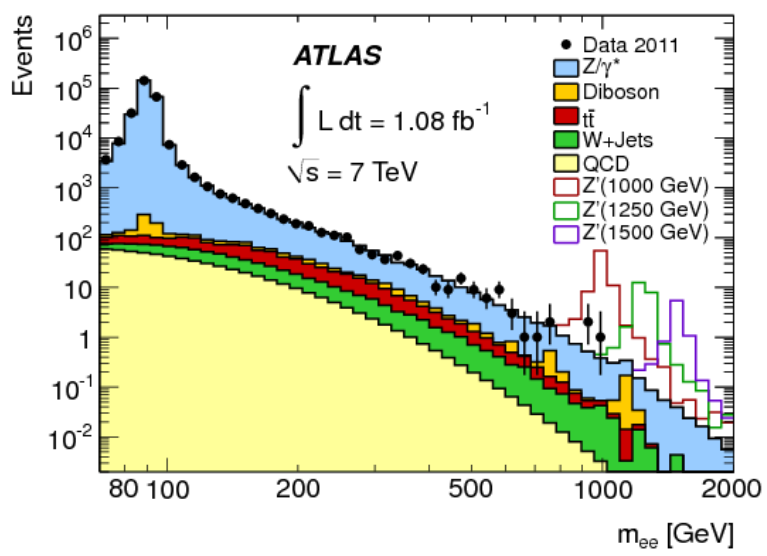

Fig.3 (ATLAS Collaboration 2011). Dielectron invariant mass $\left(m_{e e}\right)$ distribution after final selection, compared to the stacked sum of all expected backgrounds, with three example $Z_{\mathrm{SSM}}^{\prime}$ signals overlaid. The bin width is constant in $\log m_{e e}$.

(Giudice \& Sturmia 2003), making less realistic the occurrence of quantum gravity phenomena at the terascale.

Also the RS models can be tested. In such a set up the KK modes are not invisible but they should show up only as spin-2 resonances. As a result if one considers proton collisions in the $e^{+} e^{-}$channel, deviations from conventional Standard Model results in terms of narrow high mass resonances are expected (Davoudiasl, Hewett \& Rizzo 2000). Fig. 3, provided by ATLAS Collaboration (2011), shows 


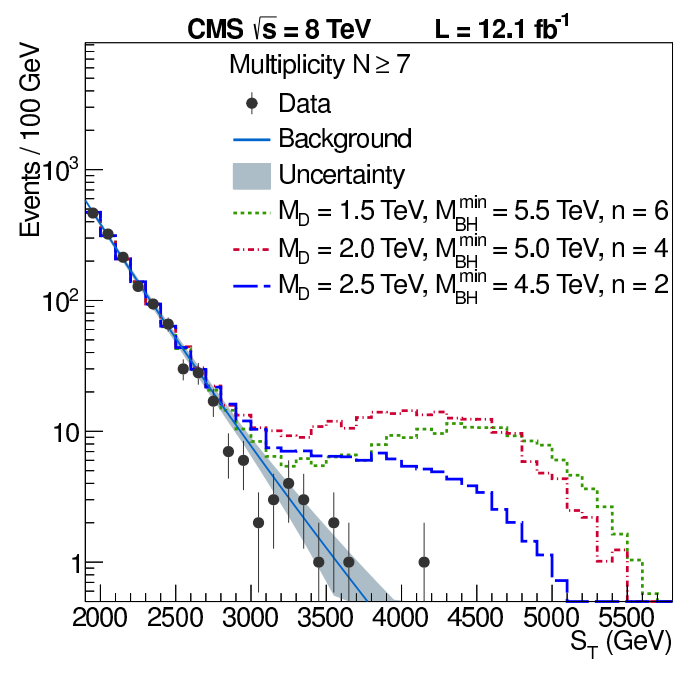

Fig. 4 (CMS Collaboration 2013). Distribution of the total transverse energy, $S_{\mathrm{T}}$, for events with multiplicity $N \geq$ 7 particles in the final state. Expected semiclassical BH signals are obtained with the BLACK MAX nonrotating BH model (Dai et al. 2008). Here, $M_{\mathrm{BH}}^{\min }$ is the minimum BH mass, $M_{D}$ is the fundamental mass in $D$ dimensions, and $n$ is the number of extra dimensions. Bottom: The 95\% confidence level (CL) lower limits on the semiclassical BH as a function of $M_{D}$, for various models. The areas below each curve are excluded by this search. The analysis is performed with CHARYBDIS BH models with or without the stable remnant.

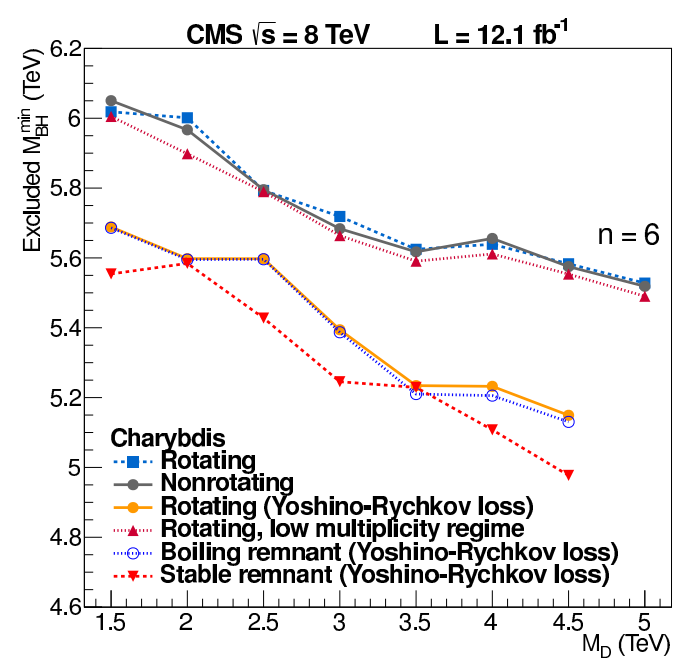

Fig. 5 (CMS Collaboration 2013). The 95\% confidence level (CL) lower limits on the semiclassical BH mass as a function of $M_{D}$, the fundamental mass in $D$ dimensions for various models. Here $n$ is the number of extra dimensions. The areas below each curve are excluded by this search. The analysis is performed with CHARYBDIS BH models with or without the stable remnant. that this is not the case: the observed invariant mass spectra are consistent with Standard Model expectations.

Finally direct searches of BHs are performed by analyzing possible high transverse energy, high multiplicity events. Also in such a case deviations from the Standard Model are not observed (see Fig. 4). Depending on the model under consideration, analyses from CMS Collaboration $(2012,2013)$ and ATLAS Collaboration (2014) for collisions at $8 \mathrm{TeV}$ exclude BHs with masses below $4.3-6.2$ $\mathrm{TeV}$ (see Fig. 5) in all cases, i.e., non-rotating, rotating BHs, with or without remnant.

\section{Conclusions}

Experimental results are negative. No BHs, no gravitons as well as no quantum gravity effects have been observed at the LHC for collision energy up to $8 \mathrm{TeV}$.

The interpretation of these findings requires a careful analysis. A possible explanation for the absence of BHs and any quantum gravity phenomenology at the terascale might be simply due to the fact that the fundamental mass exceeds the LHC design energy, i.e., $M_{\mathrm{F}}>14 \mathrm{TeV}$. If this were the case, we should rely on a "natural collider", i.e., ultra high energy cosmic rays (UHECRs), whose observed energy $E_{\mathrm{CR}}$ can reach $10^{20} \mathrm{eV}$, corresponding to $\sim 500$ $\mathrm{TeV}$ in the centre of mass frame. The problem with UHECRs is, however, related to meager fluxes. For instance at $E_{\mathrm{CR}} \sim 10^{19}$, the number of observable events is less that 1 per kilometer squared, per year. The paucity of events requires large detectors, as it happens for the Pierre Auger Observatory (PAO), which has 1600 water Cherenkov detectors distributed over 3000 square kilometers. This means that, if BHs form from the collision of UHECRs with the upper layers of Earth atmosphere, the PAO can detect 100 BHs in three years (Feng \& Shapere 2002). The current non-observations of events is used to further constraint the value of $M_{\mathrm{F}}$.

Other competitive bounds can be derived by the conjectured $\mathrm{BH}$ production in the scattering of ultrahigh energy cosmic neutrinos on nucleons in the ice or water at AMANDA/IceCube, ANTARES neutrino telescopes (Kowalski, Ringwald \& Tu 2002). This kind of analyses turn to be very sensitive since they can provide additional information about $\mathrm{BH}$ branching ratios and the various angle and energy distributions (Alvarez-Muniz et al. 2002).

The formation of BHs is certainly a leading test for terascale quantum gravity but only as a sufficient condition. The necessary condition might not be valid. In some energy regimes, terascale quantum gravity could occur without $\mathrm{BH}$ formation. If we consider spacetime geometries admitting horizon extremization and remnant formation in the Schwarzschild phase, one finds that the minimum energy for $\mathrm{BH}$ formation is $M_{\mathrm{BH}}^{\min } \equiv$ $M_{\text {remn. }} \sim 10 \mathrm{PeV}$ for $d=5$ and $M_{\mathrm{F}}=1 \mathrm{TeV}$ (Rizzo 2006, Nicolini 2008, Gingrich 2010). This is also supported by a recently proposed ghost free, singularity 
free higher derivative theory of gravity (Biswas et al. 2012). Again the horizon extremization, by deforming Hawking spectra, might lead to quite different $\mathrm{BH}$ signatures like a milder emission mostly on the brane (Koch et al. 2005; Casadio \& Nicolini 2008, Gingrich 2010, Nicolini \& Winstanley 2011). Other unconventional signatures might arise from the non-thermal decay of BHs (Calmet, Caramete \& Micu 2012, Arsene et al. 2013, Arsene et al. 2014). There exist also limitations to the $\mathrm{BH}$ production which are rather generic and independent of a specific model: It has been shown that the BH formation might be simply suppressed in order to respect wellestablished properties of particles, like the lifetime of the proton decay (Stojkovic, Starkman \& Dai 2006).

In conclusion, BHs might be extremely hard to produce and to detect. Our understanding of several aspects of terascale phenomenology is far from being complete. We recall that the majority of the literature is focused on the Schwarzschild phase, a minor part on the spin-down phase, while little is know about the balding phase. In addition there exists a variety of effects that are often underestimated, such as the role of the brane tension (Kaloper \& Kiley 2006), the role of color fields (Mavromatos \& Winstanley 2000), the validity of the approximation of quasi-stationary decay. Finally, the Planck phase is plagued by tremendous difficulties since it connects mini BHs to the problem of the formulation of a quantum theory of gravity. An observation of the Hawking temperature profile in the Planck phase or the formation of remnants could, however, provide crucial indications about the validity of current quantum gravity proposals.

Acknowledgements. This work has been supported by the grant NI 1282/3-1 of the project "Evaporation of microscopic black holes" of the German Research Foundation (DFG), by the Helmholtz International Center for FAIR within the framework of the LOEWE program (Landesoffensive zur Entwicklung WissenschaftlichÖkonomischer Exzellenz) launched by the State of Hesse and partially by the European Cooperation in Science and Technology (COST) action MP0905 "Black Holes in a Violent Universe". The authors are grateful to A. Mazumdar, O. Micu, J. Mureika and D. Stojkovic for valuable comments and references.

\section{References}

Adelberger, E. G., Heckel, B. R., Hoedl, S., Hoyle, C. D., Kapner, D. J., Upadhye, A.: 2007, PhRvL 98, 131104

Adelberger, E. G., Gundlach, J. H., Heckel, B. R., Hoedl, S., Schlamminger, S.: 2009, PrPNP 62, 102

Adler, R. J., Chen, P., Santiago, D. I.: 2001, GReGr 33, 2101

Alvarez-Muniz, J., Feng, J.L., Halzen, F., Han, T., Hooper, D.,: 2002, PhRvD 65, 124015

Antoniadis, I.: 1990, PhLB 246, 377

Antoniadis, I., Arkani-Hamed, N. Dimopoulos, S., Dvali, G. R.: 1998, PhLB 436, 257

Appelquist, T., H. -C. Cheng, H.C., B. A. Dobrescu, B. A.: 2001, PhRvD 64, 035002

Argyres, P. C., Dimopoulos, S., March-Russell, J.: 1998, PhLB 441, 96
Arkani-Hamed, N., Dimopoulos, S., Dvali, G. R.: 1998a, PhLB 429, 263

Arkani-Hamed, N., Dimopoulos, S., Dvali, G. R.: 1998b, PhRvD 59, 086004

Arkani-Hamed, N., Grossman, Y., Schmaltz, M.: 2000, PhRvD 61, 115004

Arkani-Hamed, N., Schmaltz, M.: 2000, PhRvD 61, 033005

Arsene, N., Calmet, X., Caramete, L. I., Micu, O.: 2014, APh 54, 132

Arsene, N., Caramete, L. I., Denton, P. B., Micu, O.: 2013, arXiv: 1310.2205

ATLAS Collaboration: 2011, PhRvL 107, 272002

ATLAS Collaboration: 2014, PhLB 728, 562

Banks, T., Fischler, W.: 1999, hep-th/9906038

Biswas, T., Gerwick, E., Koivisto, T., Mazumdar, A.: 2012, PhRvL 108, 031101

Bleicher, M.: 2007, EJPh 28, 509

Bleicher, M., Hofmann, S., Hossenfelder, S., Stoecker, H.: 2002, PhLB 548, 73

Bleicher, M., Nicolini, P.: 2010, JPhCS 237, 012008

Bonanno, A., Reuter, M.: 2006, PhRvD 73, 083005

Bousso, R., Hawking, S. W.: 1996, PhRvD 54, 6312

Calmet, X: 2010, MPLA 25, 1553

Calmet, X., Caramete, L. I., Micu, O.: 2012, JHEP 1211, 104

Calmet, X., Fragkakis, D., Gausmann, N.: 2012, chapter 8 in A.J. Bauer and D.G.Eiffel editors,Black Holes: Evolution, Theory and Thermodynamics Nova Publishers, New York

Carr, B.J., Hawking, S.W.: 1974, MNRAS 168, 399

Casadio, R., Nicolini, P.: 2008, JHEP 0811, 072

Casanova, A., Spallucci, E.: 2006, CQGra 23 , R45

Cavaglià, M.: 2003, IJMPA 18, 1843

Celotti, A., Miller, J. C., Sciama, D. W.: 1999, CQGrav 16, A3

Chamblin, A., Cooper, F., Nayak, G. C.: 2004, PhRvD 70, 075018

CMS Collaboration: 2012, JHEP 1204, 061

CMS Collaboration: 2013, JHEP 1307, 178

Dai, D. -C., Starkman, G., Stojkovic, D., Issever, C., Rizvi, E, Tseng, J.: 2008, PhRvD 77, 076007

Davoudiasl, H., Hewett, J. L., Rizzo, T. G.: 2000, PhRvL 84, 2080

Dimopoulos, S., Landsberg, G. L.: 2001, PhRvL 87, 161602

Emparan, R., Horowitz, G. T., Myers, R. C.: 2000, PhRvL 85, 499

Erkoca, A. E., Nayak, G. C., Sarcevic, I.: 2009, PhRvD 79, 094011

Feng, J. L.: 2003, Sci 302, 795

Feng, J. L., Shapere, A. D.: 2002, PhRvL 88, 021303

Franceschini, R., Giardino, P.P., Giudice, G. F., Lodone, P., Strumia, A.: 2011, JHEP 1105, 092

Friedland, A., Giannotti, M.: 2008, PhRvL 100, 031602

Giddings, S. B., Thomas, S. D.: 2002, PhRvD 65, 056010

Gillies, G. T.: 1997, RPPh 60, 151

Gingrich, D.M.: 2010, JHEP 1005, 022

Gogberashvili, M.: 1999, MPLA 14, 2025

Gogberashvili, M.: 2000, EL 49, 396

Gogberashvili, M.: 2002, IJMPD 11, 1635

Giudice, G. F., Strumia, A.: 2003, NuPhB 663, 377

Hawking, S. W.: 1975, CMaPh 43, 199

Hossenfelder, S.: 2005, In 'Focus on Black Hole Research', pp. 155-192, Nova Science Publishers

Hossenfelder, S., Hofmann, S., Bleicher, M., Stoecker, H.: 2002, PhRvD 66, 101502

Hossenfelder, S., Bleicher, M., Hofmann, S., Stoecker, H., Kotwal, A. V.: 2003, PhLB 566, 233

Hoyle, C. D., Kapner, D. J., Heckel, B. R., Adelberger, E. G., Gundlach, J. H., Schmidt, U., Swanson, H. E.: 2004, PhRvD 70, 042004

Isi, M., Mureika, J., Nicolini, P.: 2013, JHEP 1311, 139 
Kaloper, N., Kiley, D.: 2006, JHEP 0603, 077

Kanti, P.: 2004, IJMPA 19, 4899

Kanti, P., Winstanley, E.: 2014, arXiv:1402.3952

Koch, B., Bleicher, M., Hossenfelder, S.:, 2005, JHEP 0510, 053

Kowalski, M., Ringwald, A., Tu, H.: 2002, PhLB 529, 1

Landsberg, G.: 2002, hep-ph/0211043

Long, J.C., Chan, H.W., Churnside, A.B., Gulbis, E.A., Varney, M.C.M., Price, J.C.: 2003, Natur 421, 922

Maartens, R., 2004, LRR 7, 7

Mann, R. B., Ross, Simon F.: 1995,PhRvD 52, 2254

Mavromatos, N.E., E. Winstanley, E.: 2000, CQGra 17, 1595

Mirabelli, E.A., Schmaltz, M.: 2000, PhRvD 61, 113011

Modesto, L.: 2006, CQGra 23, 5587

Modesto, L., Moffat, J. W., Nicolini, P.: 2011, PhLB 695, 397

Mohr, P.J., Taylor, B.N., Newell, D.B.: 2012, JPCRD 41, 043109.

Mureika, J. R.: 2008, PhLB 660, 561

Mureika, J. R.: 2012, PhLB 716, 171

Mureika, J., Nicolini, P.: 2013, EPJP 128, 78

Mureika, J., Nicolini, P., Spallucci, E.: 2012, PhRvD 85, 106007

Nicolini, P.: 2008, IJMPA 24, 1229

Nicolini, P.: 2012, arXiv:1202.2102

Nicolini, P., Smailagic, A., Spallucci, E.: 2006, PhLB 632, 547

Nicolini, P., Winstanley, E.: 2011, JHEP 1111, 075

Park, S. C.: 2012, PrPNP 67, 617

Randall, L., Sundrum, R.: 1999a, PhRvL 83, 3370

Randall, L., Sundrum, R.: 1999b, PhRvL 83, 4690

Rizzo, T.G.: 2006, JHEP 0609, 021

Seiberg, N., Witten, E.: 1999, JHEP 09, 032

Shifman, M.: 2010, IJMPA 25, 199

Sprenger, M., Nicolini, P., Bleicher, M.: 2012, EJPh 33, 853

Starobinskii, A. A.: 1973, JETP 37, 28

Stojkovic, D., Starkman, G. D., Dai, D. -C.: 2006, PhRvL 96, 041303

Tangherlini, F.R.: 1963, NCimS 27, 636

Thorne, K. S., 1972, Magic Without Magic: John Archibald Wheeler, A Collection of Essays in Honor of his Sixtieth Birthday. Edited by John R. Klauder. San Francisco: W.H. Freeman, p. 231

Unruh, W. G.: 1974, PhRvD 10, 3194

Veneziano, G.: 1986, EL 2, 199V

Winstanley, E.: 2007, arXiv:0708.2656 